\title{
Study on cognitive behavioural coping of intervention and rescue personnel in toxic I flammable / explosive environments
}

\author{
Izabella Kovacs ${ }^{1, *}$, Artur George Găman ${ }^{1}$, Cosmin Ilie $^{1}$, Alin Irimia ${ }^{1}$, and Andrei Gireadă ${ }^{1}$ \\ ${ }^{1}$ National Institute for Research and Development in Mine Safety and Protection to Explosion - \\ INSEMEX Petroșani, 32-34 G-ral Vasile Milea Street, Postcode: 332047, Petroșani, Hunedoara \\ County, Romania
}

\begin{abstract}
In any given field, the psychological examination represents a prerequisite for ensuring that the work process is properly and appropriately directed towards increasing its efficiency. An important aspect of the psychological examination is to identify risk and protective factors associated with developing and maintaining emotional and behavioural problems. Special conditions resulting from emergency situations are likely to lead to physical and emotional tensions. In some intervention and rescue personnel these are accompanied by mobilization of internal resource, while in others they can generate inadequacy phenomena and symptoms of mental distress. From this perspective, stress is regarded as a result of the marked disparity between environmental requirements and the individual's response possibilities. To highlight both cognitive and behavioural coping strategies most often used by rescuers trained in NRDI INSEMEX we used two instruments, namely Strategic Approach to Coping Scale SACS and Cognitive Emotion Regulation Questionnaire CERQ. This paper displays the results of the project no. PN 164301 12, study conducted through Nucleu program, implemented with the support of NASR.
\end{abstract}

\section{Stress in the context of intervention and rescue activities}

The work of rescuers falls into the category of "hard work" in terms of effort deployed, far exceeding the average calories global consumption of workers from active work fronts. The character of hard work also comes from the fact that the occupational effort contains many high amplitude oscillations concerning the efforts deployed. The greater their duration is, the harder the work is. Rescue activities have an occasional character (they are not permanent) involving the change of daily habits from the basic profession. Also, rescuers have to perform training in conditions similar to those of the damaged area, meant to make them able to act efficiently and safely. [1-2]

The way rescue personnel cope with stressful situations depends on several variables: the circumstances of the event, personal training, pre-existing organizational stressors and

\footnotetext{
* Corresponding author: izabella.eisler@insemex.ro
} 
pre-existing personal stressors. In general, most of intervention and rescue personnel seem to favour coping responses that involve taking steps to solving problems or using logical analysis to understand the stressors related to work. Some rescuers capitalize and benefit from retreat in solitude, while others seek the company of others.

Given the short timeframe in which mental health professionals have contact with intervention and rescue personnel it is difficult to assess the effectiveness of these individual processes of adaptation. It is well known that assistance and psychological support programs are not addressed only to persons who are victims of various types of adverse events and disasters. Intervention and rescue personnel, exposed to emotional stress, may experience state of crisis as a result of professional activity, situation often found in young people with less experience. The traditional heroic role of rescuers includes the expectations that they are selfless, tireless and to some extent "superheroes". Of course, these expectations are largely unrealistic and rescue and intervention personnel are also affected by their professional activity.

Stress is inherent in many of intervention and rescue activities. Apart from the usual work-related stress in the intervention and rescue activities the following situations represent specific challenges [3]:

- Being part of a collective crisis;

- Repeated exposure to grim experiences such as handling dead bodies, dealing with multiple victims, ravishing stories of affected people;

- Performing physically difficult, strenuous or hazardous tasks;

- Lack of sleep and chronic fatigue feeling, due to the demanding tasks;

- Facing the perceived inability to ever do enough;

- Facing moral and ethical dilemmas;

- Exposure to anger and apparent lack of gratitude of the people affected;

- Exiting the home support system;

- The feeling of frustration given by the policies and decisions of superiors within the organization.

The rescuers are exposed to unusual personal demands in their desire to meet the needs of survivors from a disastrous event. The multitude of feelings associated with the intervention, such as giving first, closeness to centre of the disturbing event and not least dealing with emotionally troubled and physically hurt people, must be addressed and confronted. Rescuers, who are often poorly prepared for their own emotional reactions during rescue intervention, can be supported in several key moments in order to reduce the likelihood of developing stress-related problems. Primary intervention consists of solid information about stress and how to cope with emotional reactions in difficult situations. [4] Such information educates and prepares rescuers for detection of their own reactions and provides active options for self-help and peer support. If left unaddressed, these stressors are likely to affect the welfare and quality of rescuer's work. Thus, self-help is both an essential pre-requisite to effectively help others and a means of maintaining the capacity to continue the work of rescue. [5]

Some people have a much higher risk than normal, for developing severe symptoms of stress and lasting posttraumatic stress disorder if they have a history of:

- Exposure to trauma (serious accidents, abuse, aggression, war, business rescue);

- Medical illness or chronic psychological disorder;

- Chronic poverty, homelessness, unemployment or discrimination;

- Major stressors or recent emotional strain (such as the condition of being a single parent). 


\section{Coping strategies used by rescuers trained within NRDI INSEMEX Petrosani}

Disaster related stress can bring back to the fore memories of earlier trauma and may enhance social, economic, spiritual, psychological or medical background. In order to highlight both cognitive and behavioural ways of coping most often used by rescuers trained within NRDI INSEMEX, we have used two instruments, namely Strategic approach to coping scale SACS and Cognitive Emotion Regulation Questionnaire CERQ. The results obtained by using these instruments on a number of 38 , respectively 70 people are discussed below.

Strategic approach to coping scale SACS is a tool that assesses behavioural coping, taking into accounts the social aspects of the strategies by which one faces stressful situations. SACS highlights a person's coping strategies where they particularly manifest, respectively in a social context. This scale assesses behavioural coping strategies on the following dimensions: active-passive pro-social-antisocial, direct-indirect, including nine scales (behavioural coping strategies):

1. Active-passive dimension

- Assertive action: addressing the situation firmly, spontaneously, honestly and directly, without retreat from problems, pursuing personal interests without harming others.

- A high score for this strategy is generally associated with low emotional distress and low scores may be associated with emotional problems or psychopathological symptoms. The observation that can be made about this result is that this way of coping is little used by respondents although in the context of intervention and rescue activities, addressing stressful issues firmly, honestly and directly is a desirable coping strategy, as shown in the results achieved by applying SACS on part of the rescuers trained within INCD INSEMEX and illustrated in table 1 .

Table 1. Results for assertive action scale

\begin{tabular}{|l|c|c|c|}
\hline & Frequency & Percent & Cumulative Percent \\
\hline Under population average & 3 & 7.9 & 7.9 \\
Population average & 30 & 78.9 & 86.8 \\
Above population average & 5 & 13.2 & 100.0 \\
\hline
\end{tabular}

\section{Pro-social-antisocial dimension}

- Social networking: through which the individual joins others to face the situation together or by which the individual helps others, taking into account their needs;

- Seeking social support: the individual turn to others for help and emotional support and he consults with loved ones (family, friends) about what to do;

- These strategies to cope with stressful situations are frequently used by respondents, as can be seen from table 2, studies indicating that frequent use of these coping strategies is associated with increased levels of positive emotions.

Table 2. Results for social networking and seeking social support scales

\begin{tabular}{|l|c|c|c|c|}
\hline & \multicolumn{2}{|c|}{ Social networking } & \multicolumn{2}{c|}{ Seeking social support } \\
\hline & Frequency & Percent & Frequency & Percent \\
\hline Population average & 24 & 63.2 & 26 & 68.4 \\
Above population average & 14 & 36.8 & 12 & 31.6 \\
\hline
\end{tabular}

- Prudent action: by which the individuals take all precautions before action and carefully evaluate their options to protect themselves from any danger; 
- Instinctive action: by which the individual rests upon his own intuition and he reacts by momentary impulses to solve problems;

Table 3. Results for prudent action and instinctive action scales

\begin{tabular}{|l|c|c|c|c|}
\hline & \multicolumn{2}{|c|}{ Prudent action } & \multicolumn{2}{c|}{ Instinctive action } \\
\hline & Frequency & Percent & Frequency & Percent \\
\hline Under population average & 2 & 5.3 & 2 & 5.3 \\
Population average & 25 & 65.8 & 18 & 47.4 \\
Above population average & 11 & 28.9 & 18 & 47.4 \\
\hline
\end{tabular}

- Prudent action and instinctive action are in opposition: while the first strategy refers to careful and rational evaluation of the situation and involves consideration for others, instinctive action, which is predominant compared to prudent action among respondents in the research, situation shown in table 3 , involves impulsive action without much time to think and less consideration for others. Of course, in the context of intervention and rescue activities the prudent action strategy for coping with stressful situations seems to be preferable to instinctive action. This does not mean that this second coping strategy may not prove to be beneficial in some particular contexts.

3. Direct-indirect dimension

- Avoidance: by which the individual engages in other activities so that he no longer has to deal with the problem, by which he withdraws or expects things to solve themselves.

- According to studies, this strategy is maladaptive and is associated with high emotional distress and psychopathological symptoms. Although in lower number, as shown in table 4, this strategy is nevertheless used by rescuers who are part of the population of present research respondents.

Table 4. Results for avoidance scale

\begin{tabular}{|l|c|c|c|}
\hline & Frequency & Percent & Cumulative Percent \\
\hline Under population average & 6 & 15.8 & 15.8 \\
Population average & 23 & 60.5 & 76.3 \\
Above population average & 9 & 23.7 & 100.0 \\
\hline
\end{tabular}

- Indirect action: by which the individual manipulates the situation to make others believe that they are in control, while he solves the problem, opaquely, behind the scenes;

- Anti-social action: by which the individual pursues his interests even if they hurt others;

- Aggressive action: by which the individual act decisively, quickly, to take others by surprise in order to take control.

- As shown in table 5, the responders in the current research use these strategies just as much as the average population.

Table 5. Results for indirect action, antisocial action and aggressive action scales

\begin{tabular}{|l|c|c|c|}
\hline & Indirect action & Antisocial action & Aggressive action \\
\hline & Percent & Percent & Percent \\
\hline Under population average & 21.1 & 34.2 & 13.2 \\
Population average & 73.7 & 63.2 & 86.8 \\
Above population average & 5.3 & 2.6 & 0.00 \\
\hline
\end{tabular}

Active and pro-social coping strategies tend to be associated more often with positive emotional consequences, with low levels of anxiety and depression, while antisocial coping 
is associated with increased levels of anger and the avoidance is associated with increased levels of anxiety and depression.

Cognitive Emotion Regulation Questionnaire CERQ is designed to identify cognitive coping strategies that someone uses after experiencing negative events (thus only referring to a person's thoughts). CERQ distinguished nine different types of cognitive coping strategies:

1. Self-blaming, referring to the thoughts by which an individual blames himself for what happened. A high degree of concern regarding self-blame may be associated with psychopathological symptoms. As shown in table 6 this coping strategy is used by a significant proportion of respondents (more than half of them), situation that $\mathrm{s}$ worrisome.

Table 6. Results for self-blaming scale

\begin{tabular}{|l|c|c|c|}
\hline & Frequency & Percent & Cumulative Percent \\
\hline Very low/low & 20 & 28.6 & 28.6 \\
Under average & 1 & 1.4 & 30.0 \\
Average & 10 & 14.3 & 44.3 \\
Above average & 20 & 28.6 & 72.9 \\
High/very high & 19 & 27.1 & 100.0 \\
\hline
\end{tabular}

2. Acceptance, which refers to the thoughts by which an individual resigns himself to what happened. Although self-acceptance itself is a beneficial process for most events, a very high level of acceptance, as occurs in almost a quarter of the respondents of the present research and is shown in table 7 , can result in a negative form of resignation, meaning the inability to be able to influence events that can be associated with psychopathological symptoms.

Table 7. Results for acceptance scale

\begin{tabular}{|l|c|c|c|}
\hline & Frequency & Percent & Cumulative Percent \\
\hline Very low/low & 14 & 20.0 & 20.0 \\
Under average & 10 & 14.3 & 34.3 \\
Average & 18 & 25.7 & 60.0 \\
Above average & 11 & 15.7 & 75.7 \\
High/very high & 17 & 24.3 & 100.0 \\
\hline
\end{tabular}

3. Rumination: the individual thinks continuously of feelings and ideas associated with the event. High and very high score obtained by almost a quarter of respondents, as seen in table 8 , indicates that rumination its frequently used as a cognitive coping strategy. A high score is certainly associated with emotional problems or psychopathological symptoms; therefore the case of the responders in the current research, as it is shown in the table below, is at least worrying.

Table 8. Results for rumination scale

\begin{tabular}{|l|c|c|c|}
\hline & Frequency & Percent & Cumulative Percent \\
\hline Very low/low & 14 & 20.0 & 20.0 \\
Under average & 16 & 22.9 & 42.9 \\
Average & 8 & 11.4 & 54.3 \\
Above average & 15 & 21.4 & 75.7 \\
High/very high & 17 & 24.3 & 100.0 \\
\hline
\end{tabular}

4. Positive refocusing: the individual is thinking about other pleasant things, not the event itself. 
Table 9. Result for positive refocusing scale

\begin{tabular}{|l|l|c|c|c|}
\hline \multicolumn{1}{|c|}{} & Frequency & Percent & Cumulative Percent \\
\hline \multirow{3}{*}{} & Very low/low & 9 & 12.9 & 12.9 \\
& Under average & 3 & 4.3 & 17.1 \\
& Average & 14 & 20.0 & 37.1 \\
& Above average & 9 & 12.9 & 50.0 \\
& High/very high & 35 & 50.0 & 100.0 \\
\hline
\end{tabular}

Studies show that this strategy may have positive effects on the wellbeing of the individual, therefore the higher the scores the more we can talk about high levels of positive emotionality. Very few of our responders reported of not using this coping strategy, as can be seen in table 9, with half of them reporting to use it often or very often.

5. Refocusing on planning, by which an individual thinks about the steps they will take to face the event.

6. Positive reappraisal by which an individual thinks to assign a positive meaning to the event, in terms of personal development.

High scores on refocusing on planning and positive revaluation scales, as can be seen in table 10, show the frequent use of these coping strategies. These are functional cognitive coping strategies, but this is true only if, at the same time, something is done to solve the problem, if the individual addresses the problematic situation. If else, the score is associated with emotional problems.

Table 10. Results for refocusing on planning and positive reappraisal scales

\begin{tabular}{|l|c|c|c|c|}
\hline & \multicolumn{2}{|c|}{ Refocusing on planning } & \multicolumn{2}{c|}{ Positive reappraisal } \\
\hline & Frequency & Percent & Frequency & Percent \\
\hline Very low/low & 8 & 11.4 & 6 & 8.6 \\
Under average & 4 & 5.7 & 11 & 15.7 \\
Average & 10 & 14.3 & 12 & 17.1 \\
Above average & 18 & 25.7 & 12 & 17.1 \\
High/very high & 30 & 42.8 & 29 & 41.4 \\
\hline
\end{tabular}

7. Putting things into perspective or thoughts that minimize the seriousness of the event when compared to other events.

As shown in table 11, the responders in the current research use these strategies above the average population.

Table 11. Results for putting things into perspective scale

\begin{tabular}{|l|c|c|c|}
\hline & Frequency & Percent & Cumulative Percent \\
\hline Very low/low & 14 & 20.0 & 20.0 \\
Under average & 9 & 12.9 & 32.9 \\
Average & 14 & 20.0 & 52.9 \\
Above average & 11 & 15.7 & 68.6 \\
High/very high & 22 & 31.5 & 100.0 \\
\hline
\end{tabular}

8. Catastrophizing by which the individual emphasizes the terror caused by the event.

High scores obtained for catastrophizing, as obtained by a quarter of the respondents in our research and shown in table 12 are almost certainly associated with emotional problems or psychopathological symptoms. 
Table 12. Results for catastrophizing scale

\begin{tabular}{|l|c|c|c|}
\hline & Frequency & Percent & Cumulative Percent \\
\hline Very low/low & 12 & 17.1 & 17.1 \\
Under average & 15 & 21.4 & 38.6 \\
Average & 10 & 14.3 & 52.9 \\
Above average & 15 & 21.4 & 74.3 \\
High/very high & 18 & 25.7 & 100.0 \\
\hline
\end{tabular}

9. Blaming others by which one thinks to blame others for what happened. As shown in table 13, the responders in the current research use these strategies just as much as the average population.

Table 13. Results for blaming others scale

\begin{tabular}{|l|c|c|c|}
\hline & Frequency & Percent & Cumulative Percent \\
\hline Very low/low & 20 & 28.6 & 28.6 \\
Under average & 3 & 4.3 & 32.9 \\
Average & 24 & 34.3 & 67.1 \\
Above average & 14 & 20.0 & 87.1 \\
High/very high & 9 & 12.9 & 100.0 \\
\hline
\end{tabular}

Cognitive coping strategies are defined as cognitive strategies for emotion regulation which involve cognitive regulation of emotional responses to events resulting in worsening individual emotions. These coping strategies refers to rather constant styles of coping cope with negative events but at the same time they can be influenced, e.g. changed through learning or forgotten, for example through various intervention programs or personal experiences.

As stress as a whole cannot be removed, the main objective in coping with stress is to feel particularly eustress in the detriment of distress and to prevent distress to degenerate into dysfunctional behaviours. In the context of intervention and rescue activities, dysfunctional behaviours such as alteration of decision-making capacity, mistakes, impulsive and disorganized behaviour, behaviour paralysis or panic can lead to further damage and loss of human lives.

Therefore, we find that respondents, intervention and rescue personnel trained within NRDI INSEMEX, use both adaptive strategies and strategies whose use is associated with emotional problems and even psychopathology. On one hand being aware that they use a strategy or another (with benefits and drawbacks involved) and, on the other hand psychological training interventions and personal development can help individuals learn the use of adaptive strategies to the detriment of those associated with emotional distress.

\section{Conclusions}

The work of rescuers falls into the category of "hard work" in terms of effort deployed, the plurality, diversity and especially the nature of the tasks involved in the intervention and rescue activities. Rescue activities have an occasional character; they are not permanent, involving the change of daily habits from the basic profession. As well as performing training in conditions similar to those of the damaged area, which make the rescuers able to act effectively and safely, intervention and rescue personnel, that during or after intervention, experiences or is direct witness of violence, injuries, extreme destruction, 
prolonged exposure to fatigue, bad weather, starvation, toxic contamination is at high risk for developing severe symptoms of stress and lasting problems regarding recovery.

The results attained by applying SACS and CERQ instruments on a total of 38, respectively 70 individuals indicate that intervention and rescue personnel trained within NRDI INSEMEX uses both adaptive strategies and strategies whose use is associated with emotional problems and even psychopathology. On one hand being aware that they use a strategy or another (with benefits and drawbacks involved) and, on the other hand psychological training interventions and personal development can help individuals learn the use of adaptive strategies to the detriment of those associated with emotional distress.

The training process of intervention and rescue personnel within NRDI INSEMEX would capitalize on a psychological training programme, to help individuals learn the use of adaptive coping strategies.

\section{References}

1. C. Ilie, Research on aspects related to structuring exercise and psychosocial training to optimize the process of intervention and rescue personnel in toxic / flammable / explosive environments (NRDI INSEMEX Research study, 2012)

2. D. Pupăzan, C. Ilie, Intervention and rescue in toxic / flammable / explosive environments , (NRDI INSEMEX Training material for rescuers, 2014)

3. https://mhpss.net/?get=58/1354772532-Mod06CommBasedPSSSupport-HelpingtheHelpers.pdf

4. http://www.ceep.ca/resources/Guterman2005_Psychological_Preparedness for_Disaster.pdf

5. http://www.ptsd.va.gov/professional/treatment/early/disaster-rescue-response.asp 